\title{
shRNA knockdown of DNA helicase ERCC6L expression inhibits human breast cancer growth
}

\author{
JUAN LIU, JING SUN, QIAN ZHANG and ZHAOCHONG ZENG \\ Department of Radiotherapy, Zhongshan Hospital, Fudan University, Xuhui, Shanghai 200032, P.R. China
}

Received December 28, 2017; Accepted June 26, 2018

DOI: $10.3892 / \mathrm{mmr} .2018 .9317$

\begin{abstract}
Breast cancer is a heterogeneous disease with a high degree of diversity with regards to tumor histological stage and molecular subtypes. These heterogeneous characteristics determine the risk of disease progression and therapeutic resistance. Understanding tumor heterogeneity is of primary concern to identify and develop novel and specific potential targets for diagnosis and therapy. The present study analyzed 106 paired breast cancer tissues from The Cancer Genome Atlas and demonstrated that excision repair cross-complementation group 6 like (ERCC6L), a newly discovered DNA helicase, was overexpressed in $91.51 \%(97 / 106)$, unchanged in $7.54 \%$ $(8 / 106)$ and decreased in $0.94 \%(1 / 106)$ of breast cancer samples. A short hairpin RNA ERCC6L lentivirus was constructed to investigate the role of ERCC6LR in cancer. First, a Celigo Image Cytometry system was used to detect MDA-MB-231 cell growth number following transfection with shERCC6L-lentivirus or NC-lentivirus and it was identified that the growth number of fluorescent MDA-MB-231 cells post-transduction with shERCC6L-lentivirus was decreased compared with the cells transduced with NC-lentivirus. Then, the effect of knockdown of ERCC6L expression on the cell cycle distribution and apoptosis was to analyzed using fluorescence-activated cell sorting (FACS). The FACS data demonstrated that knockdown of ERCC6L expression levels in MDA-MB-231 cells significantly increased $S$ phase population but decreased the $\mathrm{G}_{1}$ and $\mathrm{G}_{2} / \mathrm{M}$ phase populations compared with the NC group. The apoptosis rate of MDA-MB-231 cells post-transduction with shERCC6L-lentivirus for 5 days was increased to $12.16 \pm 0.146 \%$ compared with the negative control
\end{abstract}

Correspondence to: Professor Zhaochong Zeng, Department of Radiotherapy, Zhongshan Hospital, Fudan University, 180 Fenglin Road, Xuhui, Shanghai 200032, P.R. China

E-mail: zeng.zhaochong@zs-hospital.sh.cn

Abbreviations: TCGA, The Cancer Genome Atlas; ERCC6L, excision repair cross-complementation group 6 like; ER, estrogen receptor; HER2, human epidermal growth factor receptor 2; PR, progesterone receptor

Key words: ERCC6L, breast cancer, proliferation, apoptosis rate $(4.86 \pm 0.204 \%)$. These functional studies demonstrated that knockdown of ERCC6L expression levels in MDA-MB-231 cells significantly inhibited breast cancer cell proliferation, disturbed cell cycle distribution and induced apoptosis in vitro. These findings suggested that ERCC6L, which is highly expressed in breast cancer, acts as an oncogene, is involved in breast cancer development and may serve as a novel molecular target for the treatment of breast cancer.

\section{Introduction}

Breast cancer is the most common cancer and the second leading cause of cancer-associated mortality among women worldwide, with $\sim 1.7$ million cases and 521,900 fatalities occurring in 2012 (1). Breast cancer is a highly heterogeneous type of cancer; this heterogeneity is evident with regards to tumor morphology and at the transcriptome and proteome levels $(2,3)$. It is difficult to identify specific and sensitive diagnostic and therapeutic targets; therefore, understanding the molecular and cellular mechanisms of tumor heterogeneity that are relevant to the diagnosis, prognosis and treatment of breast cancer is a primary research concern $(4,5)$.

For the study of breast cancer heterogeneity, it has been acknowledged that breast cancer is categorized into three basic therapeutic groups, based on the expression levels of estrogen receptor (ER), human epidermal growth factor receptor 2 (HER2; also termed ERBB2) and progesterone receptor (PR), which can also be used to classify breast cancer phenotype heterogeneity. Recently, a study from Sweden reported a comparative analysis of ER, PR, HRR2 and Ki67 status between the primary tumor and corresponding relapse and found that the discordance of four receptors status was 14.2, 39.6, 9.6 and $36.3 \%$, respectively. Loss of ER or PR in the relapse resulted in a significantly increased risk of mortality [hazard ratio (HR) 3.62; 95\% confidence interval (CI), 1.65-7.94] and (HR 2.34; 95\% CI, 1.01-5.47) compared with patients with stable ER or PR positive tumors (6). It is also established that there is no available effective treatment available for triple-negative breast cancer, which does not express ER, HER2 or PR. Therefore, the identification of specific tumor targets for breast cancer is required.

The development of genome sequencing technology has resulted in more objective ways to identify tumor heterogenetic genes, such as variant molecular subtypes and critical mutations genes, in order to aid understanding of the molecular 
function and mechanisms of the disease (7). For example, phosphatidylinositol-4,5-bisphosphate 3-kinase catalytic subunit alpha (PIK3CA) and p53 have been identified as the two most frequently mutated genes in breast cancer (8). When the oncogenic PIK3CA-H1047R mutant is expressed at physiological levels in basal cells, it induces the formation of luminal ER-positive/PR-positive tumors in animal models, whereas its expression in luminal cells gives rise to luminal $\mathrm{ER}^{+} \mathrm{PR}^{+}$tumors or basal-like ER-PR tumors (9). In addition, Koren et al (10) demonstrated that concomitant expression of PIK3CA-H1047R and deletion of p53 accelerates tumor development, inducing more aggressive mammary tumors. These findings provided information regarding the molecular mechanisms underlying tumor heterogeneity and suggested the potential therapeutic applications of blocking key oncogenes, such as PIK3CA. There are currently no tumor-specific genes or proteins that have been identified for all breast cancer subtypes; therefore, the present study aimed to analyze 'breast cancer-associated genes' using high-throughput molecular profiling data, in order to identify potential genes for diagnosis and therapy. Genomic data for breast cancer were obtained from The Cancer Genome Atlas (TCGA), and it was observed that excision repair cross-complementation group 6 like (ERCC6L) was highly expressed in $91.51 \%$ of breast cancers, although the role of ERCC6L in breast cancer remains unclear. Functional studies were also performed to further confirm that ERCC6L may act as an oncogene involved in tumor development and progression, thus suggesting that ERCC6L may be a potential target for breast cancer treatment.

\section{Materials and methods}

Data acquisition. For breast cancer, there are 1,097 available data samples which contains 106 pairs of data. In the present study, the gene expression data were from 106 breast cancer samples (18 were stage I, 61 stage II and 27 stage III-IV) and were downloaded from TCGA (http://cancergenome.nih.gov; RNA-Seq Version and RNA-Seq Version 2). All data standardization sets were processed using the Trimmed Mean of M-values normalization method.

Cell culture. The breast cancer cell line MDA-MB-231 was obtained from the Institute of Biochemistry and Cell Biology of the Chinese Academy of Science (Shanghai, China). MDA-MB-231 cells were maintained in Dulbecco's modified Eagle's medium (DMEM; HyClone, GE Healthcare Life Sciences, Logan, UT, USA) supplemented with $10 \%$ fetal bovine serum (FBS; Gibco; Thermo Fisher Scientific, Inc., Waltham, MA, USA) and $1 \%$ penicillin/streptomycin (Gibco; Thermo Fisher Scientific, Inc.) in a $5 \% \mathrm{CO}_{2}$ incubator at $37^{\circ} \mathrm{C}$.

Lentivirus construction and cell transduction. All lentiviral constructs were prepared by Shanghai GeneChem Co., Ltd. (Shanghai, China). Briefly, the ERCC6L-specific short hairpin (sh)RNA (shERCC6L) and negative control (NC) shRNA were designed and synthesized by Shanghai GeneChem Co., Ltd. and their sequences were as follows: 5'-TCATGCCAACCAATCTTAT-3' and 5'-TTCTCCGAA
CGTGTCACGT-3', respectively. These fragments were inserted into the $A g e \mathrm{I} / E c o R I$ site of a lentivirus linearized hU6-MSC-CMV-puro ${ }^{\mathrm{r}}$ vector (GV115, $7.5 \mathrm{~kb}$; GeneChem Co., Ltd.) carrying green fluorescent protein (GFP). For lentivirus packaging, approximately $24 \mathrm{~h}$ before transfection, $5 \times 10^{6}$ HEK 293 cells were seeded into $10 \mathrm{~cm}$ tissue culture plates in $10 \mathrm{ml}$ of growth medium and incubated at $37^{\circ} \mathrm{C}$ in $5 \% \mathrm{CO}_{2}$ overnight. The cells were $70 \%$ confluent at the time of transfection. Then, $2 \mathrm{~h}$ prior to transfection, the medium was replaced with serum-free DMEM. Then the transfection medium (15 $\mu \mathrm{g}$ pHelper plasmids, $10 \mu \mathrm{g}$ pHelper 2.0 plasmids, $20 \mu \mathrm{g}$ lentivirus packing vector GV115 and Lipofectamine ${ }^{\circledR} 2000$ (volumes as recommended by the manufacturer, Invitrogen; Thermo Fisher Scientific, Inc.) was respectively added into a sterilized tube, then mixed and adjusted to $1 \mathrm{ml}$, and incubated at room temperature for $15 \mathrm{~min}$. Finally, the transfection medium was added to the HEK 293 cells and incubated at $37^{\circ} \mathrm{C}$ for $6 \mathrm{~h}$. Then the medium was removed and replaced with new culture medium and culture continued for an additional 48-h. The lentiviral supernatants were harvested and filtered through a $0.45 \mu \mathrm{m}$ low protein binding filter to remove cellular debris. shRNA lentiviruses were concentrated by ultracentrifugation $(2 \mathrm{~h}$ at $50,000 \mathrm{x} \mathrm{g}$ ) and subsequently purified on a sucrose $20 \%$ gradient $(2 \mathrm{~h}$ at $46,000 \mathrm{x} \mathrm{g}$ ) for future use. The virus titer of shERCC6L and shCtrl was $4 \times 10^{8} \mathrm{TU} / \mathrm{ml}$ and $5 \times 10^{8} \mathrm{TU} / \mathrm{ml}$, respectively.

For cell transduction, $2 \times 10^{6}$ MDA-MB-231 cells per well were seeded in a 6 well-plate overnight and then infected with lentiviruses [multiplicity of infection (MOI) 20]. Following incubation for $72 \mathrm{~h}$ at $37^{\circ} \mathrm{C}$ in a $5 \% \mathrm{CO}_{2}$ incubator, most cells expressed green fluorescent protein (GFP) under fluorescence microscopy. When the infection rate of MDA-MB-231 cells reached more than $70 \%$, the cells were used in the following experiments. Reverse transcription-quantitative polymerase chain reaction (RT-qPCR) and western blotting were used to determine the effectiveness of the shRNA on ERCC6L knockdown.

Cell Proliferation. Following transfection with shERCC6L or shCtrl lentivirus for $72 \mathrm{~h}, \mathrm{MDA}-\mathrm{MB}-231$ cells were seeded at a density of $2 \times 10^{3}$ cells ( $200 \mu \mathrm{l} /$ per well) in 96-well plates and cultured at $37^{\circ} \mathrm{C}$ in a $5 \% \mathrm{CO}_{2}$ incubator for 5 days. A Celigo Image cytometer (Nexcelom Bioscience, Lawrence, MA) was used to detect the growth number of MDA-MB-231 cells at fixed time points.

RNA extraction and $R T-q P C R$. Cells were collected at $48 \mathrm{~h}$ post-transduction and total RNA was extracted using TRIzol ${ }^{\circledR}$ reagent (Invitrogen; Thermo Fisher Scientific, Inc.). RT was performed with random nucleotide primers using an M-MLV RT system (Promega Corporation, Madison, WI, USA), according to the manufacturer's protocol. RT-qPCR with gene-specific primers was performed using GoTaq qPCR master mix (Promega Corporation). GAPDH was used as the internal control. The real-time PCR program consisted of $30 \mathrm{sec}$ at $95^{\circ} \mathrm{C}, 40$ cycles of $5 \mathrm{sec}$ at $95^{\circ} \mathrm{C}, 1 \mathrm{~min}$ at $60^{\circ} \mathrm{C}$ and a dissociation stage at the end of the run from 60 to $95^{\circ} \mathrm{C}$. It was performed in a Roche LightCycler480 Real-Time PCR system. The relative mRNA expression levels were 
determined by the cycle quantification $(\mathrm{Cq})$ normalized against GAPDH using the $2^{-\Delta \Delta C q}$ formula (11). The primers used were as follows: ERCC6L, forward 5'-CTCTGGCTT GCTACTTTATCGAG-3', reverse 5'-TGCATCAAACAT ACCGGAAAGG-3'; GAPDH, forward 5'-TGACTTCAA CAGCGACACCCA-3', and reverse 5'-TGCATCAAACAT ACCGGAAAGG-3'.

Western blotting. Cells were lysed with radioimmunoprecipitation assay buffer (RIPA; cat. no. P0013B; Beyotime Institute of Biotechnology, Haimen, China) and proteins were quantified using a Bicinchoninic Protein Assay kit (cat. no. P0010S, Beyotime Institute of Biotechnology). A total of 20-50 $\mu \mathrm{g}$ total protein was separated by $8 \%$ SDS-PAGE and subsequently transferred onto polyvinylidene difluoride membranes (cat. no. IPVH00010; EMD Millipore, Billerica, MA, USA). Following blocking in TBST (Tris-buffered saline with $0.5 \%$ Tween-20) which contained 5\% non-fat milk for $60 \mathrm{~min}$ at room temperature, the membranes were incubated with the primary antibody overnight at $4^{\circ} \mathrm{C}$ on a shaker. The primary antibody includes mouse anti-ERCC6L (1:1,000; cat. no. SAB1407576; Sigma-Aldrich; Merck KGaA, Darmstadt, Germany) and mouse anti-GAPDH (1:2,500; cat. no. sc-47724; Santa Cruz Biotechnology, Inc., Dallas, TX, USA). Following washing with $1 \%$ TBST buffer for $10 \mathrm{~min}$ 3 times, the membranes were incubated with the HRP-linked goat anti-mouse IgG secondary antibodies (1:2,000 dilution; cat. no. 7060; Cell Signaling Technology, Inc., Danvers, MA, USA). Following incubation in enhanced chemiluminescence solution (cat. no. M3121; Thermo Fisher Scientific, Inc.) according to the manufacturer's protocols. The proteins on the membranes were detected using Bio-Rad Universal Hood III, and analyzed by Image $\mathrm{Lab}^{\mathrm{TM}}$ software version 2.0 (Bio-Rad Laboratories, Inc., Hercules, CA, USA).

Apoptosis assay and cell cycle analysis. For apoptosis analysis, $1 \times 10^{6} / \mathrm{ml}$ MDA-MB-231 cells were harvested at $48 \mathrm{~h}$ post-transduction, and the level of apoptosis was determined using an Annexin V apoptosis detection kit (eBioscience; Thermo Fisher Scientific, Inc.), according to the manufacturer's protocol. As Annexin V is an allophycocyanin (APC) dye, the apoptosis analysis was performed on a fluorescence-activated cell sorting (FACS) machine (EMD Millipore) with an APC channel. For cell cycle analysis, 1x10\%/ml MDA-MB-231 cells were collected following transduction and fixed with cold $70 \%$ alcohol at $4^{\circ} \mathrm{C}$ overnight. Alcohol was removed and cells were washed with cold PBS. Cells were stained with propidium iodide solution (Sigma-Aldrich; Merck KGaA) containing $20 \mu \mathrm{g} / \mathrm{ml}$ RNase A (Fermentas; Thermo Fisher Scientific, Inc.) and incubated at room temperature for $30 \mathrm{~min}$. Following filtration with a nylon mesh filter, cell cycle analysis was performed on a fluorescence-activated cell sorting (FACS) machine (EMD Millipore). DNA content analysis was performed with ModFit LT software (Verity Software House, Inc., Topsham, ME, USA) to calculate cell cycle phase.

Statistical analysis. Each experiment was performed $\geq 3$ times. All data are expressed as the means \pm standard deviation. Unless otherwise noted, the differences of continuous variables between two groups were analyzed by Student's t-test or

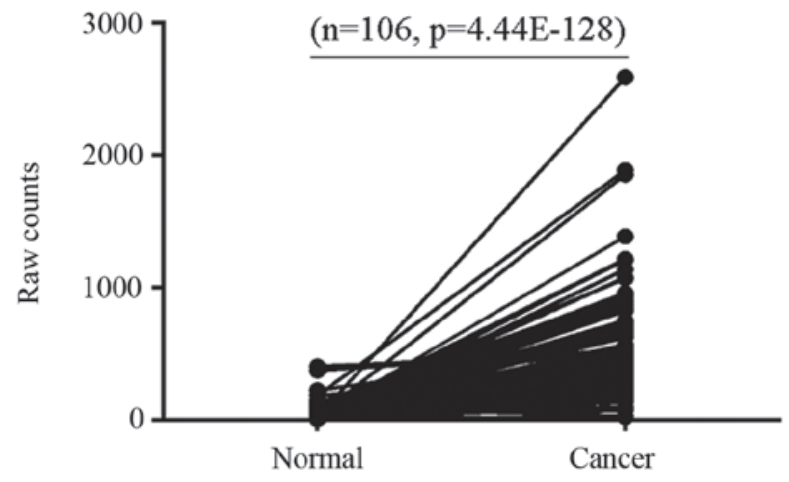

Figure 1. ERCC6L is highly expressed in breast cancer tissues. The expression levels of ERCC6L in 106 paired breast cancer samples from The Cancer Genome Atlas were normalized by the Trimmed Mean of M-values method. ERCC6L was highly expressed in 91.51\% (97/106) of breast cancer samples compared with the matched controls. ERCC6L, excision repair cross-complementation group 6 like.

Wilcoxon rank-sum test. All analyses were performed using SPSS software version 17.0 (Chicago, IL, USA). P $<0.05$ was considered to indicate a statistically significant difference.

\section{Results}

ERCC6L is overexpressed in breast cancer tissues. The present study analyzed data from 106 paired breast cancer samples at different pathological stages from TCGA and demonstrated that ERCC6L expression was higher in 91.51\% (97/106), unchanged in $7.54 \%(8 / 106)$ and decreased in $0.94 \%(1 / 106)$ of breast cancer samples compared with in matched controls (Fig. 1). These results suggested that abnormal expression of ERCC6L may be involved in breast cancer development.

Effects of ERCC6L silencing breast cancer cells. In order to investigate the role of ERCC6L in breast cancer, the present study constructed and evaluated the knockdown effects of a shERCC6L-lentivirus. As demonstrated in Fig. 2A, fluorescence microscopy revealed that the transduction efficiency of the GFP-containing shERCC6L-lentivirus in MDA-MB-231 cells following transfection for $72 \mathrm{~h}$ was $>80 \%$, and the virus MOI was 20. Data from RT-qPCR revealed that the knockdown effect of the shERCC6L-lentivirus in MDA-MB-231 cells was $>91.50 \%$ (Fig. 2B) and the western blotting results simultaneously indicated that the shERCC6L-lentivirus decreased the expression levels of ERCC6L in MDA-MB-231 cells (Fig. 2C). These data suggested that the shERCC6L lentivirus was successfully constructed.

ERCC6L ShRNA inhibits breast cancer cell proliferation. To determine the effects of ERCC6L on the proliferation of MDA-MB-231 cells, a Celigo Image Cytometry system was used to detect MDA-MB-231 cell growth post-transduction with shERCC6L-lentivirus or NC-lentivirus, at a specific time-point. As demonstrated in Fig. 3A, the number of fluorescent MDA-MB-231 cells post-transduction with shERCC6L-lentivirus was decreased compared with cells transduced with NC-lentivirus. In addition, the number (Fig. 3B) and growth (Fig. 3C) of MDA-MB-231 cells was markedly decreased compared with the NC cells. These findings indicated that 
A

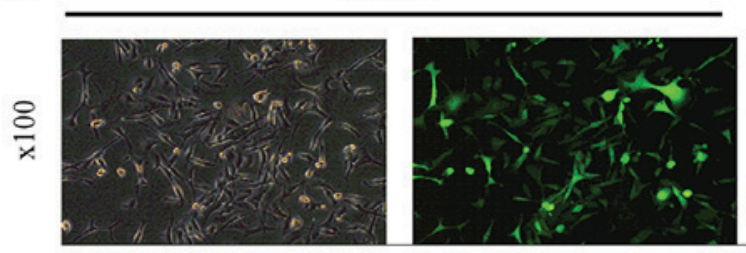

B

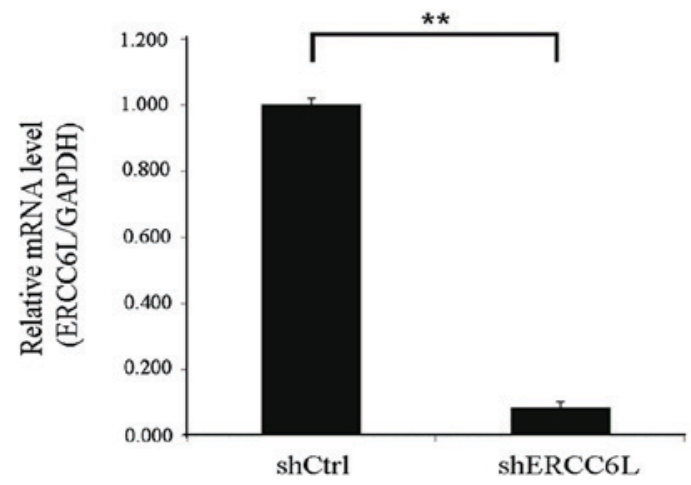

shERCC6L

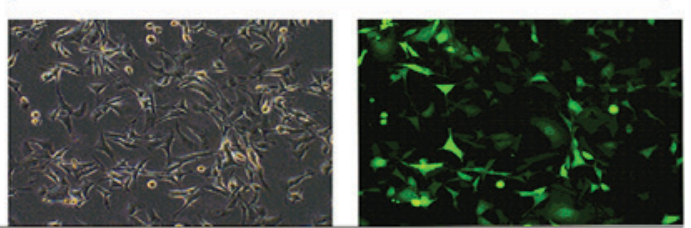

C

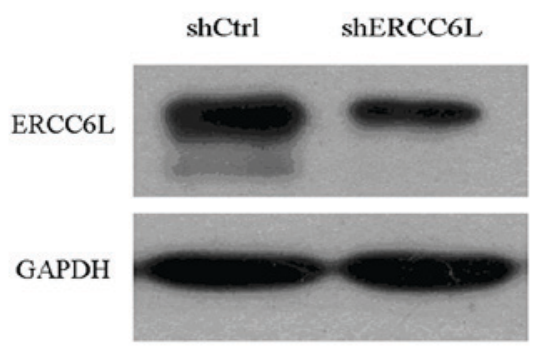

Figure 2. Effects of knockdown of ERCC6L on MDA-MB-231 cells transduced with shERCC6L-lentivirus for 3 days, as evaluated by RT-qPCR and western blotting. (A) Fluorescence images of MDA-MB-231 cells following transduction with shERCC6L-lentivirus for 3 days. (B) mRNA expression levels of ERCC6L in MDA-MB-321 cells following ERCC6L silencing, as detected by RT-qPCR. (C) Protein expression levels of ERCC6L in MDA-MB-231 cells following transduction with shERCC6L-lentivirus or negative control (shCtrl), as analyzed by western blotting. ** P<0.01. ERCC6L, ERCC6L, excision repair cross-complementation group 6 like; RT-qPCR, reverse transcription-quantitative polymerase chain reaction; sh, short hairpin RNA.
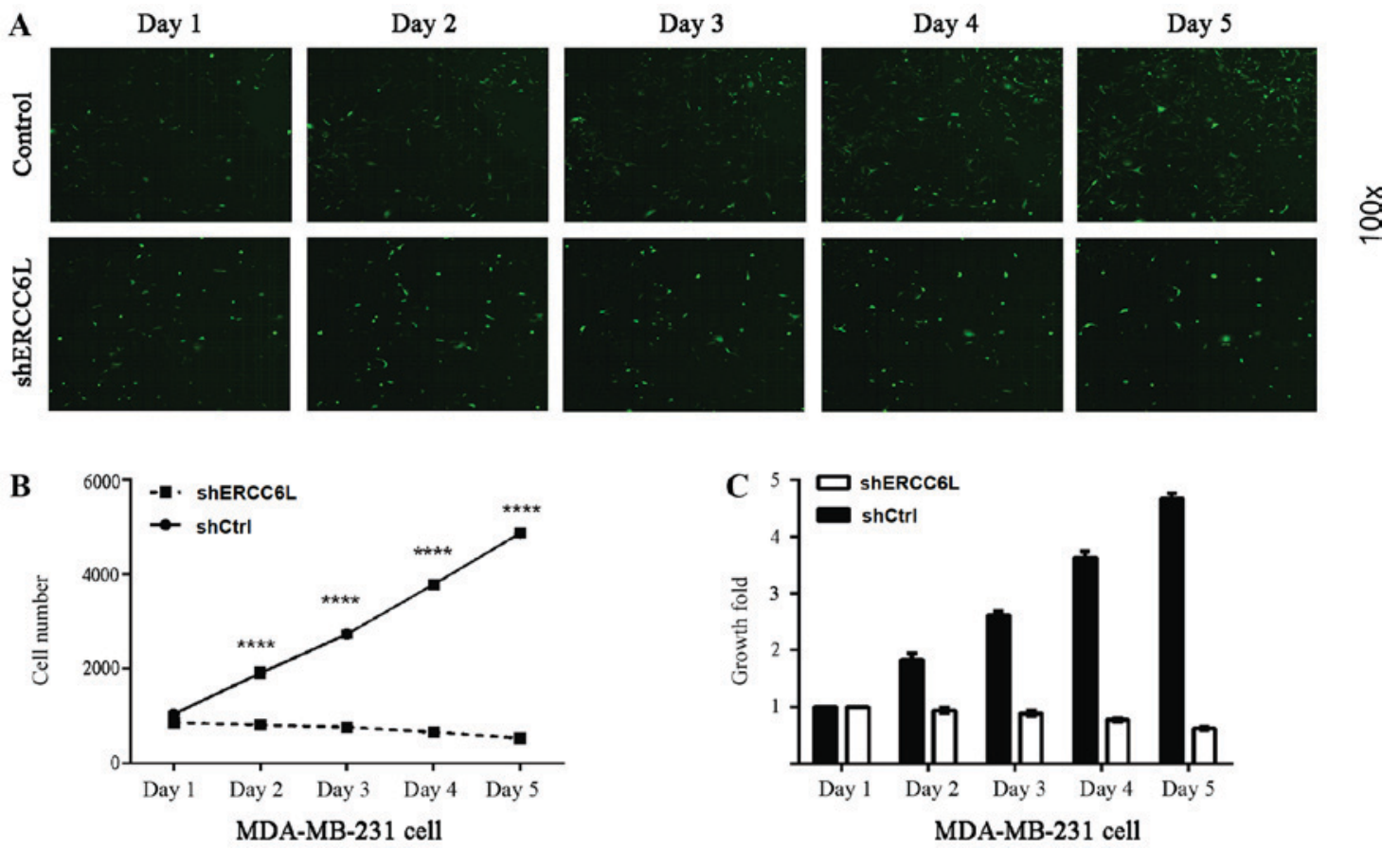

Figure 3. Silencing ERCC6L significantly inhibits MDA-MB-231 cell proliferation. (A) Celigo Image Cytometry system detected the number of MDA-MB-231 fluorescent cells following transduction with shERCC6L-lentivirus at various time points. Cells were visualized at x100 magnification. (B) Quantification of cell numbers. shCtrl was used as negative control. ${ }^{* * * *} \mathrm{P}<0.00001$ (C) Fold-change of cell growth following transduction with ERCC6L-lentivirus or negative control (shCtrl) compared with at day 1. ERCC6L, ERCC6L, excision repair cross-complementation group 6 like; sh, short hairpin RNA.

silencing ERCC6L may suppress breast cancer cell proliferation.

ERCC6L affects the cell cycle distribution of MDA-MB-231 cells. It is well known that cell proliferation and apoptosis are linked by cell cycle regulation $(12,13)$, therefore the present study aimed to identify whether ERCC6L affects the cell cycle. Cell cycle analysis indicated that the $\mathrm{S}$ phase population of MDA-MB-231 was increased post-transduction with the shERCC6L-lentivirus, whereas G1 and G2/M phase populations were decreased compared with in the NC group $(\mathrm{P}<0.01$; Fig. 4). These results suggested that ERCC6L may 
A

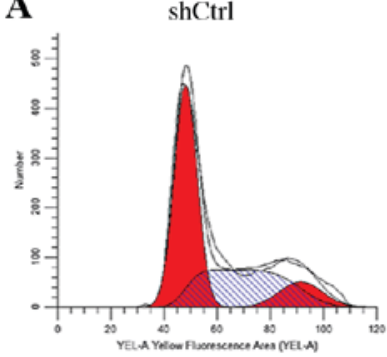

shERCC6L

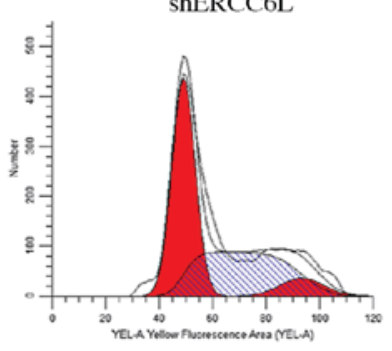

B

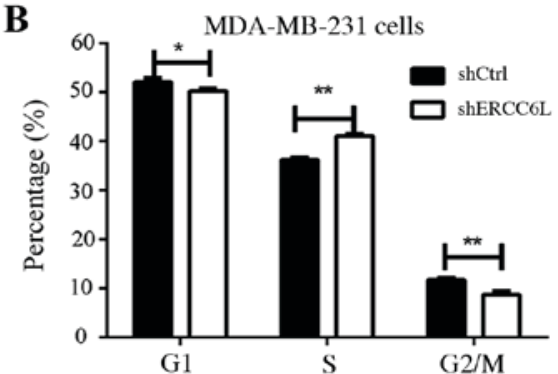

Figure 4. Effects of silencing ERCC6L expression levels on MDA-MB-231 cell cycle progression. (A) MDA-MB-231 cells transduced with shERCC6L-lentivirus or negative control for 2 days were harvested and analyzed by fluorescence-activated cell sorting. (B) Cell cycle distribution was quantified. Data are presented as the means \pm standard deviation of triplicate independent experiments. ${ }^{*} \mathrm{P}<0.05,{ }^{* *} \mathrm{P}<0.01$. ERCC6L, ERCC6L, excision repair cross-complementation group 6 like; sh, short hairpin RNA.

A

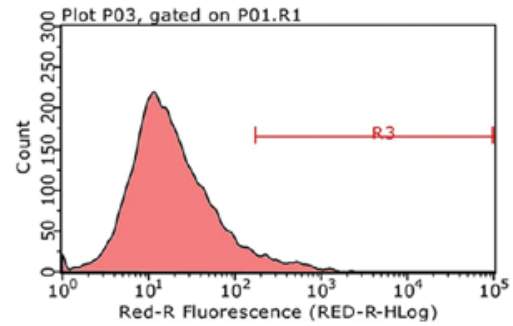

$\operatorname{shCtr}$

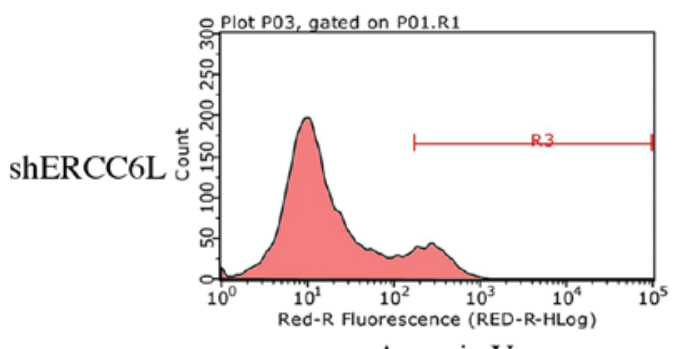

B

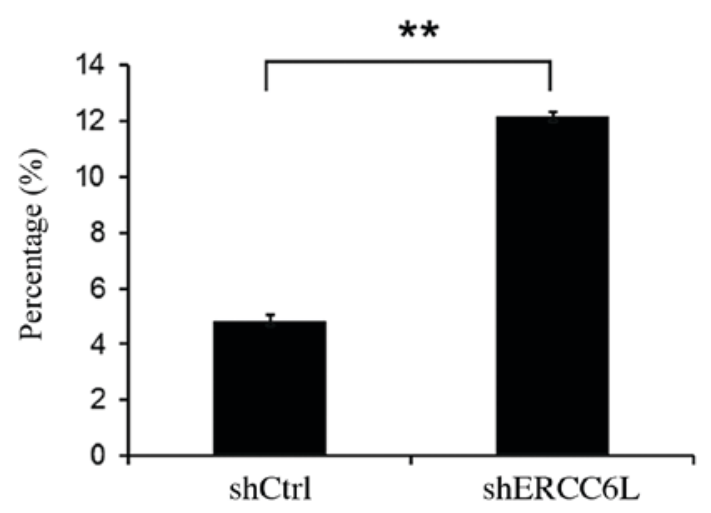

Figure 5. Silencing ERCC6L induces MDA-MB-231 cell apoptosis. (A) MDA-MB-231 cells post-transduction with shERCC6L-lentivirus for 2 days were harvested and stained with Annexin V and measured by fluorescence-activated cell sorting. (B) Apoptosis was quantified. Data are presented as the means \pm standard deviation of triplicate independent experiments. ${ }^{* *} \mathrm{P}<0.01$. ERCC6L, ERCC6L, excision repair cross-complementation group 6 like; sh, short hairpin RNA.

be involved in the DNA synthesis process in breast cancer cells.

Knockdown of ERCC6L expression induces MDA-MB-231 cell apoptosis. In addition to cell cycle analysis, the present study investigated whether knockdown of ERCC6L expression in MDA-MB-231 cells induced cell apoptosis. FACS was used to analyze the apoptosis rate of MDA-MB-M231 cells which were passaged and cultured for 2 days following infection for $72 \mathrm{~h}$. FACS analysis demonstrated that the apoptosis rate of shERCC6L-MDA-MB-231 cells cultured for 2 days was increased to $12.16 \pm 0.1462 \%$ compared with the negative control rate $(4.86 \pm 0.204 \%$; Fig. 5$)$. These findings demonstrated that knockdown of ERCC6L expression levels suppressed MDA-MB-231 cell growth by affecting the cell cycle and by inducing apoptosis.

\section{Discussion}

To the best of the authors' knowledge, breast cancer is the first type of cancer that has been reported to exhibit phenotypic heterogeneity, and tumor heterogeneity is currently one of the most highly investigated areas in cancer research (14). Tumor heterogeneity is typically observed in cellular morphology, and is present at the transcriptome and proteome levels, resulting in difficulties in identifying effective diagnostic markers and therapeutic treatments (4). The development of genome-wide technology has provided the opportunity to comprehensively understand disease mechanisms (15). Elucidation of the molecular and cellular mechanisms underlying tumor heterogeneity that are relevant to the development of treatment resistance is a major area of research.

The present study conducted an in-depth data analysis with high-throughput molecular profiling data to identify potential and useful targets for the development of novel therapies. Briefly, data from 106 paired breast cancer samples at different pathological stages from TCGA were analyzed, and it was demonstrated that ERCC6L was highly expressed in $91.51 \%$ (97/106), unchanged in $7.54 \%$ $(8 / 106)$ and decreased in $0.94 \%(1 / 106)$ of breast cancer samples compared with matched controls. This result was 
in agreement with that reported by $\mathrm{Pu}$ et al (16). Previous studies also suggested that ERCC6L is highly expressed in several types of tumor, including bladder, kidney, oral and gastric cancers (17-20). Previous studies also indicated that ERCC6L genetic polymorphisms are associated with cancer susceptibility. Abbasi et al (19) reported that laryngeal cancer risk is associated with genetic polymorphisms in ERCC5, ERCC6 and RAD23 homolog B, nucleotide excision repair protein. Liu et al (20) also revealed that the DNA repair gene ERCC6 rs1917799 polymorphism is associated with gastric cancer risk in the Chinese population. It has also been identified that ERCC6 polymorphisms are associated with susceptibility to oral, lung, bladder and colorectal cancers (21-23). These findings suggested that ERCC6L may act as an oncogene involved in tumor progression, and may be considered a potential target to aid the efficient diagnosis and development of therapies against breast cancer.

ERCC6L is a DNA helicase, also termed polo-like kinase 1-interacting checkpoint 'helicase', and is an embryonic development-associated protein (12). A previous study reported that ERCC6L is overexpressed in the embryonic heart, brain and other tissues; however, it is rarely expressed in adult tissues (24). Another study also demonstrated that ERCC6L is critical to embryonic development (17). DNA helicases are important components of DNA replication, recombination and repair in all eukaryotes and bacteria; in response to aberrant ERCC6L functioning, DNA damage and genetic instability are induced, which in turn may enhance cancer development (19). Pu et al (16) reported that ERCC6L knockdown induces G0/G1 cell cycle arrest and inhibits cell proliferation, but does not increase apoptosis of MCF-7 breast cancer cells. However, in the present study, it was demonstrated that downregulation of ERCC6L in breast cancer cells using a shRNA-containing lentivirus, caused cell growth inhibition, apoptosis and cell phase distribution. The difference between these findings may be due to cell line differences or culture conditions.

In conclusion, the present results suggested that ERCC6L may act as an oncogene involved in breast cancer development and progression. However, further in vitro and in vivo studies are required to fully reveal the effects and the underlying mechanisms of ERCC6L on tumorigenesis, treatment resistance and tumor differentiation. Silencing of ERCC6L inhibited breast cancer growth in vitro, thus indicating that ERCC6L may be a potential target for the treatment of breast cancer; however, this requires validation.

\section{Acknowledgements}

The authors thank Xiaoyun Shen from the Sir Run Run Shaw hospital affiliated to Zhejiang University School of Medicine for providing general support and $\mathrm{Xu}$ Liu from Zhongshan Hospital, Fudan University for providing assistance in writing the manuscript.

\section{Funding}

This study was supported by a grant from the Youth Science Foundation of Zhongshan Hospital, Fudan University (grant no. 2017ZSQN35).

\section{Availability of data and materials}

All data generated or analyzed during this study are included in this published article.

\section{Authors' contributions}

JL performed the experiments, analyzed the data, and drafted the manuscript. JS participated in the design of the study, helped to perform the analysis, provided valued discussions and helped to draft the manuscript. QZ helped in the sequence alignment. ZZ contributed to the conception and design of the study.

\section{Ethics approval and consent to participate}

Not applicable.

\section{Patient consent for publication}

Not applicable.

\section{Competing interests}

The authors declare that they have no competing interests.

\section{References}

1. Torre LA, Siegel RL, Ward EM and Jemal A: Global cancer incidence and mortality rates and trends-an update. Cancer Epidemiol Biomarkers Prev 25: 16-27, 2016.

2. Johann DJ, Rodriguez-Canales J, Mukherjee S, Prieto DA Hanson JC, Emmert-Buck M and Blonder J: Approaching solid tumor heterogeneity on a cellular basis by tissue proteomics using laser capture microdissection and biological mass spectrometry. J Proteome Res 8: 2310-2318, 2009.

3. Polyak K: Heterogeneity in breast cancer. J Clin Invest 121: 3786-3788, 2011.

4. Park SY, Gönen M, Kim HJ, Michor F and Polyak K: Cellular and genetic diversity in the progression of in situ human breast carcinomas to an invasive phenotype. J Clin Invest 120: 636-644, 2010.

5. Beca F and Polyak K: Intratumor heterogeneity in breast cancer. Adv Exp Med Biol 882: 169-189, 2016.

6. Karlsson E, Appelgren J, Solterbeck A, Bergenheim M, Alvariza $\mathrm{V}$ and Bergh J: Breast cancer during follow-up and progression - A population based cohort on new cancers and changed biology. Eur J Cancer 50: 2916-2924, 2014.

7. Badve S and Gökmen-Polar Y: Tumor heterogeneity in breast cancer. Adv Anat Pathol 22: 294-302, 2015.

8. Bai X, Zhang E, Ye H, Nandakumar V, Wang Z, Chen L, Tang C, Li J, Li H, Zhang W, et al: PIK3CA and TP53 gene mutations in human breast cancer tumors frequently detected by ion torrent DNA sequencing. PLoS One 9: e99306, 2014.

9. Van Keymeulen A, Lee MY, Ousset M, Brohée S, Rorive S, Giraddi RR, Wuidart A, Bouvencourt G, Dubois C, Salmon I, et al: Reactivation of multipotency by oncogenic PIK3CA induces breast tumour heterogeneity. Nature 525: 119-123, 2015.

10. Koren S, Reavie L, Couto JP, De Silva D, Stadler MB, Roloff T, Britschgi A, Eichlisberger T, Kohler H, Aina O, et al: PIK3CA(H1047R) induces multipotency and multi-lineage mammary tumours. Nature 525: 114-118, 2015.

11. Livak KJ and Schmittgen TD: Analysis of relative gene expression data using real-time quantitative PCR and the 2(-Delta Delta C(T)) method. Methods 25: 402-408, 2001.

12. King KL and Cidlowski JA: Cell cycle regulation and apoptosis. Annu Rev Physiol 60: 601-617, 1998.

13. Alenzi FQ: Links between apoptosis, proliferation and the cell cycle. Br J Biomed Sci 61: 99-102, 2004.

14. Foote FW Jr and Stewart FW: A histologic classification of carcinoma of the breast. Surgery 19: 74-99, 1946. 
15. McGranahan $\mathrm{N}$ and Swanton $\mathrm{C}$ : Biological and therapeutic impact of intratumor heterogeneity in cancer evolution. Cancer Cell 27: 15-26, 2015.

16. Pu SY, Yu Q, Wu H, Jiang JJ, Chen XQ, He YH and Kong QP: ERCC6L, a DNA helicase, is involved in cell proliferation and associated with survival and progress in breast and kidney cancers. Oncotarget 8: 42116-42124, 2017.

17. Baumann C, Körner R, Hofmann $\mathrm{K}$ and Nigg EA: PICH, a centromere-associated SNF2 family ATPase, is regulated by Plk1 and required for the spindle checkpoint. Cell 128: 101-114, 2007.

18. Lin Z, Zhang X, Tuo J, Guo Y, Green B, Chan CC, Tan W, Huang Y, Ling W, Kadlubar FF, et al: A variant of the Cockayne syndrome B gene ERCC6 confers risk of lung cancer. Hum Mutat 29: 113-122, 2008.

19. Abbasi R, Ramroth H, Becher H, Dietz A, Schmezer P and Popanda O: Laryngeal cancer risk associated with smoking and alcohol consumption is modified by genetic polymorphisms in ERCC5, ERCC6 and RAD23B but not by polymorphisms in five other nucleotide excision repair genes. Int J Cancer 125: $1431-1439,2009$
20. Liu JW, He CY, Sun LP, Xu Q, Xing CZ and Yuan Y: The DNA repair gene ERCC6 rs1917799 polymorphism is associated with gastric cancer risk in Chinese. Asian Pac J Cancer Prev 14: 6103-6108, 2013.

21. Chang $\mathrm{CH}$, Chiu CF, Wang HC, Wu HC, Tsai RY, Tsai CW, Wang RF, Wang CH, Tsou YA and Bau DT: Significant association of ERCC6 single nucleotide polymorphisms with bladder cancer susceptibility in Taiwan. Anticancer Res 29: 5121-5124, 2009.

22. Ma H, Hu Z, Wang H, Jin G, Wang Y, Sun W, Chen D, Tian T, Jin L, Wei Q, et al: ERCC6/CSB gene polymorphisms and lung cancer risk. Cancer Lett 273: 172-176, 2009.

23. Ramaniuk VP, Nikitchenko NV, Savina NV, Kuzhir TD, Rolevich AI, Krasny SA, Sushinsky VE and Goncharova RI: Polymorphism of DNA repair genes OGG1, XRCC1, XPD and ERCC6 in bladder cancer in Belarus. Biomarkers 19: 509-516, 2014.

24. Yin Y, Tang L, Zhang J, Tang B and Li Z: Molecular cloning and gene expression analysis of Ercc61 in Sika Deer (Cervus nippon hortulorum). PLoS One 6: e20929, 2011. 